\title{
Les inspecteurs primaires et les pratiques d'enseignement du français aux monolingues bretons sous la III République
}

Primary inspectors and methods of teaching French to monolingual Bretons under the 3rd Republic

Die Elementarschulinspektoren und die Unterrichtspraxis des

Französischunterrichts für einsprachige Bretonen während der Dritten Republik

\section{Yannick Le Marec}

\section{(2) OpenEdition \\ Journals}

Édition électronique

URL : https://journals.openedition.org/histoire-education/2602

DOI : 10.4000/histoire-education.2602

ISSN : 2102-5452

\section{Éditeur}

ENS Éditions

Édition imprimée

Date de publication : 20 octobre 2013

Pagination : 5-32

ISBN : 978-2-84788-498-2

ISSN : 0221-6280

\section{Référence électronique}

Yannick Le Marec, «Les inspecteurs primaires et les pratiques d'enseignement du français aux monolingues bretons sous la IIIe République », Histoire de l'éducation [En ligne], 137 | 2013, mis en ligne le 20 octobre 2014, consulté le 20 mai 2021. URL : http://journals.openedition.org/histoire-education/ 2602 ; DOI : https://doi.org/10.4000/histoire-education.2602 


\title{
Les inspecteurs primaires et les pratiques d'enseignement du français aux monolingues bretons sous la III ${ }^{\mathrm{e}}$ République
}

\author{
Yannick LE MAREC
}

Les raisons et les méthodes d'enseignement, qui ont fait du français une langue nationale et ont vu consécutivement l'affaiblissement des langues régionales, constituent un problème ouvert depuis deux siècles et un domaine de recherche largement défriché par les historiens ${ }^{1}$. Un colloque tenu à Montpellier en 2007 permet de se faire une idée de l'étendue des recherches, de la diversité des situations et, surtout, de nuancer les approches de la question, souvent travaillée trop exclusivement dans ses dimensions idéologiques et politiques. Dans sa conclusion, Patrick Cabanel estime qu'en matière de relation entre l'école et la langue, "on a peut-être trop volontiers considéré la seule "offre" venue d'en haut, en l'occurrence de l'État [... et que] la demande montée du bas, ou l'absence de demande, devraient être mieux prise en compte»². Demande sociale versus politique étatique? L'objectif de cet article est de montrer que, sur une question précise, celle des méthodes d'enseignement du français aux jeunes enfants monolingues bretons, la réponse est à travailler entre ces deux

1 Jean-François Chanet, L'École républicaine et les petites patries, Paris, Aubier, 1996. L'auteur apporte des précisions utiles sur la situation du Sud-Ouest ou de l'Alsace. Pour d'autres exemples régionaux récemment étudiés, voir Fanch Broudic, La pratique du breton de l'ancien régime à nos jours, Rennes, Presses universitaires de Rennes, 1995; Pascal Ottavi, Le bilinguisme dans l'école de la République? Le cas de la Corse, Ajaccio, Albiana, 2008.

2 Patrick Cabanel, dans Hervé Lieutard, Marie-Jeanne Verny (coord.), L'école française et les langues régionales, $\mathrm{XIX}^{e}-\mathrm{XX}{ }^{e}$ siècles, Montpellier, Presses universitaires de la Méditerranée, 2007, p. 349. 
échelons du local et du national. Les grandes déclarations, les principes et les programmes de l'école républicaine ont dû être mis en pratique, donc adaptés d'une part à ce que l'administration elle-même appelle le "milieu local", mais aussi à ce que nous pourrions appeler aujourd'hui les compétences pédagogiques des instituteurs. Notre étude s'intéresse à ces cadres intermédiaires de l'Instruction publique que furent les inspecteurs primaires, parcourant en tous sens leur circonscription, au contact des autorités locales et des maitres, dans leur effort de traduction des exigences du ministère à travers des modèles pédagogiques utilisables dans les classes.

Les réformes de l'école primaire à la fin du XIX ${ }^{\mathrm{e}}$ siècle affirment le rôle central de l'apprentissage du français et font de cette question un problème pédagogique majeur. On n'entrera pas ici dans les détails de ces réformes, qui ont été étudiées par André $\mathrm{Chervel}^{3}$. Vu d'en haut, l'apprentissage du français doit permettre de lutter contre les "patois" qui servent l'Église, garantir l'unité du pays et former l'esprit des enfants. Le français est un outil d'éducation intellectuelle et de transformation sociale et politique. Ces perspectives nécessitent de penser autrement la nature et les méthodes de son enseignement. Avec le plan d'études des écoles primaires de 1882 et l'arrêté de 1887, il devient enseignement "élémentaire" de la langue et de la littérature nationales. Nouvelle conception, nouveaux exercices, c'est à un rééquilibrage de toute la didactique du français que l'on assiste au cours des années 1880-18904. Dans ce cadre, il est essentiel de pouvoir accompagner les changements, d'en expliquer les fondements et de convaincre les acteurs de l'existence d'un autre cadre pédagogique. Comment faut-il enseigner le français aux jeunes bretons monolingues entrant à l'école? Cette question, avant d'être posée aux maîtres, est d'abord le problème professionnel de l'inspecteur.

Les sources de ce travail sont les rapports annuels des inspecteurs primaires et les dossiers individuels des instituteurs conservés aux archives départementales du Morbihan ${ }^{5}$. Nous avons dépouillé les dossiers libres à la consultation des lettres A à L, soit environ la moitié du lot. Nous n'avons retenu, sauf exceptions,

3 André Chervel, L'enseignement du français à l'école primaire. Textes officiels, t. 2 : 1880-1939, Paris, INRP/Économica, 1995, p. 9.

4 André Chervel, Histoire de l'enseignement du français du XVII ${ }^{2}$ au XX ${ }^{e}$ siècle, Paris, Retz, 2008, p. 706.

5 Aux Archives départementales du Morbihan, les dossiers individuels sont classés selon le sexe des enseignants. Nous avons choisi les instituteurs, en suivant Fanch Broudic (1995), sur leur plus grand attachement à la langue bretonne car il paraissait intéressant d'observer si cette caractéristique avait eu des effets pédagogiques dans l'apprentissage du français. 
que les dossiers des enseignants des circonscriptions de Pontivy ${ }^{6}$ et de Lorient situées à l'ouest de la ligne Sébillot ${ }^{7}$. Dans cette première sélection, nous avons conservé un corpus de 147 rapports concernant 38 instituteurs exerçant en cours préparatoire, cours élémentaire ${ }^{8}$ ou classe unique afin de focaliser notre attention sur les "commençants", les élèves bretons monolingues qui entrent à l'école sans connaître la langue française. Les rapports s'étalent sur la période 1885-1932. C'est assurément, du point de vue de l'école et de la langue, une période d'intenses changements dans les campagnes de l'ouest du Morbihan, comme dans toute la Bretagne bretonnante puisque, comme le montre Fanch Broudic, c'est le moment de la transition linguistique. Il souligne le rôle, non exclusif, de l'école comme "outil sans lequel l'apprentissage généralisé de la langue française aurait été impossible"; tout en ajoutant qu'après la Grande Guerre, l'école en français "a correspondu globalement aux attentes de la population ${ }^{9}$. Ce moment est donc un temps de mutation lorsqu'il s'agit de considérer la croissance de la demande sociale de français. Il l'est aussi au regard des débats sur l'enseignement de la langue française et des moyens pédagogiques pour y parvenir. Nous y reviendrons.

Ces rapports annuels et d'inspections individuelles permettent d'apercevoir les traces des choix opérés dans ces moments de réorganisation pédagogique et d'amener des éclairages sur l'acte d'écriture des rapports d'inspection. L'inspecteur primaire est d'abord un rouage administratif qui assure la transmission des informations et le contrôle du travail des instituteurs ${ }^{10}$. Le rapport d'inspection est à la fois l'outil et le résultat de cette activité. Il semble s'adresser directement au maître inspecté qui le signe, mais il est aussi écrit en direction de l'inspecteur d'académie qui, en posant son visa, vérifie la conformité du travail de l'inspecteur primaire dans la mise œuvre des objectifs fixés. Au-delà de ces caractéristiques, il est possible de considérer autrement le rapport d'inspection, de le voir comme

6 Cet ouest du Morbihan a fait l'objet d'un travail monographique très utile sur le XIX ${ }^{\mathrm{e}}$ siècle : André Quintric, Le développement de la scolarisation et la vie des écoliers en centre Bretagne au XIX ${ }^{e}$ siècle. L'exemple de la région de Pontivy, Rennes, CRDP, 1988.

7 Frontière linguistique tracée par le folkloriste Paul Sébillot qui établit, en 1886, la limite du breton parlé à l'ouest d'une ligne qui va de Batz-sur-Mer en Loire-Inférieure à Plouha dans les Côtes-duNord. Dans le Morbihan, cette ligne passe par Muzillac et quitte le département à l'est de Pontivy. Quelques rapports concernent néanmoins les circonscriptions de Plœrmel et de Vannes.

8 Le CP concerne (a priori) les élèves de 6-7 ans, et le CE ceux de 7 à 9 ans.

9 Fanch Broudic, La pratique du breton..., op. cit., p. 391-392.

10 Sur les inspecteurs, en Bretagne, voir le travail de Gilbert Nicolas, "Entre attaches régionales et service de l'État : les inspecteurs primaires de l'Ouest au XIX ${ }^{\mathrm{e}}$ siècle", Annales de Bretagne et des Pays de l'Ouest, t. 109, nº 2002 et plus généralement, Jean Ferrier, Les inspecteurs des écoles primaires, 1835-1995, Paris, L'Harmattan, 1997. 
un encodage de l'activité du maître à partir de principes inférés des nouvelles normes de l'enseignement du français dans la recherche tâtonnante d'un modèle pédagogique. En effet, si la lecture du bulletin de visite ou du rapport d'inspection ne nous apporte qu'une connaissance très indirecte de l'activité des maîtres et des élèves, elle nous permet d'établir les traces d'une activité de production d'un discours normatif. Elle contient les marques d'une tâche en train d'être définie en même temps qu'elle se fonde en prescription. Cette condition est nécessaire pour comprendre le caractère parfois erratique du discours inspectoral, son aspect non fini, ses intuitions ou ses remarques constructives qui émergent d'une visite. Ce que l'inspecteur primaire note, c'est-à-dire ce qu'il retient, les gestes qui l'intéressent et les mots qu'il utilise pour les nommer ou en proposer d'autres, semble souvent à distance dans une vision partielle, et évidemment partiale, du travail de l'enseignant. Ces mots constituent le matériau des catégories et normes en cours de définition. Autrement dit, notre approche du rapport d'inspection rejoint celle d'Aude Béliard et Émilie Biland quand elles considèrent que «l'action des institutions s'exerce moins par une violence monolithique que par toute une série d'opérations de codage $\|^{11}$. Il faut entendre pour nous le découpage de l'activité, la sélection de quelques objets comme la façon de lire, l'usage du tableau, ou par la suite le rapport à l'élève, et donc la catégorisation de l'activité du maitre au regard de ces objets de travail, le diagnostic que l'inspecteur primaire établit. On peut aussi y voir l'exercice d'une violence quand on se rend compte que, chez certains maîtres, ces objets et les catégories de l'inspecteur n'ont pas de sens pour eux.

Voici le cas de Noël C. que l'inspecteur décrit en 1885 comme "un frère récemment défroqué, peu instruit, peu intelligent, manquant d'initiative mais animé de bonnes intentions" dans sa classe de 57 élèves. L'inspecteur caractérise son activité en disant que "l'instituteur ne sait pas expliquer la lecture et en ce qui concerne les autres exercices, il s'en tient servilement aux indications du livre». Deux ans plus tard, lors d'une autre inspection, il est dit que "les leçons orales sont rares et mal faites", "la lecture est encore trop rapide et monotone". "Les devoirs sont assez bien choisis sauf ceux de grammaire, toujours trop nombreux. Les dictées pas corrigées ». De fait, "les élèves parlent fort difficilement; la moitié ne comprennent pas le français " ${ }^{12}$.

11 Aude Béliard, Émilie Biland, "Enquêter à partir des dossiers personnels. Une ethnographie des relations entre institutions et individus ", Genèses, nº 70, mars 2008, p. 106-119.

12 Archives départementales du Morbihan [désormais AD], 889W2067. 
Les précisions sur les capacités du maître sont à mettre en rapport avec le fait qu'il n'est pas passé par l'école normale; ce qui est censé expliquer ses méthodes que les rapports annuels caractérisent comme étant globalement "routinières et peu intelligentes", "machinales" dans le domaine de la lecture. On y lit donc la critique que les inspecteurs font des "exercices chéris des vieux maitres d'autrefois" : lecture collective des élèves, "monotone" et "chantonnante", longues analyses écrites accompagnées de "récitation inintelligente de règles non comprises", "dictées plus longues encore "13. Cette opération de codage des compétences de l'instituteur établit ainsi, en creux, d'autres nécessités qui ont dû déstabiliser Noël C. : faire parler les élèves, les exercer au langage, c'est-à-dire au maniement du français dans des leçons orales qui sont d'abord et avant tout des "exercices de langage». En réalité, par ces critiques virulentes, c'est toute une conception du métier qui est proposée au changement. Bien sûr, l'enseignant doit être formé. Mais surtout, ce sont d'autres principes et d'autres règles d'action qui doivent organiser le métier ${ }^{14}$. Cet article propose donc de s'interroger sur les stratégies et moyens mis en œuvre par les inspecteurs pour engager cette transformation.

\section{I - Un constat d'inefficacité}

À la fin des années 1880, même si les débats restent ouverts sur le rôle du breton dans l'apprentissage du français ${ }^{15}$, sur le terrain, la situation semble tranchée. Seul le français est en usage à l'école, comme le notent les règlements intérieurs, selon une formulation qui évite de nommer et de caractériser le breton. Pourtant, il ne suffit pas d'édicter des règlements, il faut aussi favoriser de nouvelles pratiques. Les écoles normales sont bien en charge de porter ces réformes et de former les nouveaux instituteurs capables de les mettre en œuvre. Mais cela ne suffit pas et les inspecteurs primaires sont à la recherche de solutions. Le tableau qu'ils dressent de la situation du Morbihan les incite à faire vite.

13 AD Morbihan, T1012, rapport annuel de l'inspecteur d'académie, 1888.

14 Les notions de principes et de règles d'action sont utilisées en référence à la didactique professionnelle. Voir Pierre Pastré, "Quelques réflexions sur l'organisation de l'activité enseignante", Recherche et formation, $\mathrm{n}^{\circ}$ 56, 2007, p.81-93.

15 Laurent Puren, "Pédagogie, idéologie et politique linguistique. L'exemple de la méthode Carré appliquée à la francisation de la Bretagne à la fin du XIX siècle", Glottopol, nº 1, janvier 2003, p. 33-53; Hervé Terral, "L'école française en Bretagne au regard de la Revue pédagogique», in Hervé Lieutard, Marie-Jeanne Verny (coord.), L'école française et les langues régionales, $\mathrm{XIX}^{e}-\mathrm{XX}{ }^{e}$ siècles, op. cit., p. 201-215; Pierre Boutan, "Langue nationale et langues régionales à l'école : le débat politique en 1925", Mots, nº6, décembre 1999, p. 29-48. 
Certes, le Morbihan n'a pas des problèmes fondamentalement différents des autres départements français ${ }^{16}$. Mais le programme de 1882 est réellement ambitieux. Ne projette-t-il pas de fonder une culture - celle d'un enseignement français - capable de rivaliser avec la culture classique? Les difficultés sont réelles et valent pour tous. André Chervel observe que, quinze ans après le lancement de la réforme, certains comme le grammairien Brelet en font un bilan mitigé : "l'enseignement du français est nouveau : sa méthode n'est pas encore bien assise». Ce qui fait dire à l'historien :

"Le problème est beaucoup plus grave qu'une question de formation professionnelle comme cela a pu être le cas dans les années 1830 par le renouvellement du corps des instituteurs. C'est un problème de méthode : elles n'existent pas, il faut les inventer et il faut du même coup se débarrasser d'une partie des méthodes traditionnelles ${ }^{\prime 17}$.

Si les problèmes de l'enseignement de la langue française peuvent donc être compris avec le recul comme un problème général qui aurait valu pour tout le territoire, on peut se demander quelle en était la perception en Bretagne bretonnante. À lire les écrits des inspecteurs du primaire, elle s'avère complexe, nécessitant de prendre en compte des éléments du contexte local et le débat sur l'usage des langues vernaculaires, avant d'interroger les méthodes.

Les rapports de l'inspecteur d'académie dont nous disposons ne cachent pas les difficultés de l'enseignement du français. Si les propos se veulent d'abord nuancés ("les instituteurs se rendent bien compte aujourd'hui du but à atteindre dans l'enseignement de la langue maternelle "18 ou bien, "quoique l'on trouve encore malheureusement dans nos écoles des enfants ne comprenant pas la question posée la plus élémentaire qui leur est adressée en français, il y a lieu, malgré tout, de constater de sensibles progrès ${ }^{19}$ ), la répétition année après année pendant vingt ans de ce discours nous laisse entendre les difficultés mais aussi les résistances. Les inspecteurs eux-mêmes envisagent plusieurs types de difficultés.

16 L'enquête de Jean-François Chanet souligne la force et la persistance des "patois " et analyse le cas de l'Alsace au lendemain de la Grande Guerre avec une population massivement germanophone. Jean-François Chanet, L'école républicaine..., op. cit., p. 265-272.

17 André Chervel, Histoire de l'enseignement du français, op. cit., p. 63.

18 AD Morbihan, T1012, rapport de l'inspecteur d'académie au préfet, 1888.

19 Ibid., 1890. 


\section{1 - Un milieu défavorable}

Les difficultés sont d'abord dues à ce que ces administrateurs nomment le "milieu ". En effet, si globalement l'enseignement du français s'avère une tâche complexe dans tous les arrondissements du Morbihan, il l'est davantage dans les arrondissements de Lorient et de Pontivy, situés à l'ouest de la ligne Sébillot. L'inspecteur primaire de Lorient écrit, dans son rapport pour l'année 1887, à propos de la lecture et de la langue française : "c'est ici la partie la plus délicate et la plus difficile de la tâche du maitre, surtout dans notre circonscription $"^{20}$. Selon l'inspecteur d'académie, "pour l'enseignement du français, l'arrondissement de Pontivy est bien inférieur aux trois autres. L'usage général de la langue bretonne dans cette partie du Morbihan est évidemment un obstacle sérieux aux progrès scolaires $»^{21}$. Les enfants "ne parlent que breton dans leur famille $"^{22}$ et le maintien de cette langue dans la vie courante est considéré comme la cause essentielle de la faible avancée du français. "Rien d'étonnant donc si l'enseignement de la langue française donne des résultats très faibles " ${ }^{23}$. Cette situation perdure, et jusqu'à la Première Guerre mondiale, on retrouve dans les rapports des mentions identiques. Ainsi, en 1900, "le français est encore la partie la plus faible de nos campagnes : nos enfants pensent en breton et traduisent ensuite leurs pensées en français »"24 et encore en 1907, «Français : enseignement ingrat puisque le breton est la langue maternelle et que dans la presque totalité des villages pas un enfant ne parle français quand il arrive en classe ${ }^{25}$.

Cette situation de diglossie, marquée par un usage du breton dans la vie quotidienne et du français à l'école n'existe cependant pas de manière homogène et l'inspecteur primaire de Pontivy précise qu'il existe des exceptions, dans les villes chefs-lieux de canton, ce qui est tout à fait convergent avec les études historiques sur les langues en Bretagne. Fanch Broudic, qui a étudié l'évolution de la pratique sociale du breton, montre que la période 1863-1952 est celle pendant laquelle on passe de $86 \%$ de monolingues bretons, essentiellement dans le monde rural, à leur quasi disparition (6\%). Il considère que c'est aux alentours de la Première

20 Ibid., rapport de l'inspecteur primaire de Lorient, 1887.

21 Ibid., rapport annuel de l'inspecteur d'académie du Morbihan, 1888.

22 AD Morbihan, 889W2080.

23 Ibid., rapport de l'inspecteur primaire de Pontivy, 1888.

24 AD Morbihan, T1048, rapport de l'inspecteur primaire de Pontivy, 1900.

25 AD Morbihan, T1049, rapport de l'inspecteur primaire de Pontivy, 1907. 
Guerre mondiale que se produit le premier basculement ${ }^{26}$. Cependant, Fanch Broudic évoque la situation de la population bretonne en général, pas celle des jeunes enfants, en particulier. Si l'on considère les entrants au cours préparatoire (CP), notre corpus nous permet de constater la lenteur de cette évolution marquée par des situations variables dans le cadre scolaire. Encore en 1918, dans l'école de village de Silfiac, à quelques kilomètres à l'ouest de Pontivy, l'inspecteur constate toujours des "mauvais résultats en raison de l'ignorance du français qui est le fait de tous les élèves du cours préparatoire, très nombreux par ailleurs $»^{27}$. En 1923, à Ploërdut, l'inspecteur fait le lien entre "conditions de vie des enfants" et enseignement en "milieu breton" 28 .

Les témoignages de trois enseignants nés dans la première moitié du XX $\mathrm{X}^{\mathrm{e}}$ siècle, pris dans le département voisin du Finistère, permettent la comparaison et éclairent cette tendance lente. Leurs remarques sont aussi intéressantes pour comprendre les situations auxquelles sont confrontées les instituteurs. PierreJakez Hélias, né en 1914, ne parle que le breton quand il entre au cours préparatoire autour de 1920. "Au début, nous avons beau faire, nous entendons du breton dans les paroles de la maîtresse des petits. Ou plutôt, nous essayons, vaille que vaille, de reconnaitre dans la suite des sons qu'elle émet des mots bretons "29. Pour Jean Rohou, c'est en 1939 et il ne sait "dire en français que oui, non, papa, maman et sans doute Madame pour désigner l'institutrice $\aleph^{30}$. PierreMarie Mallégol est entré au CP vers 1947. Il ne comprend pas un mot de français et se sent très handicapé dans ses premiers moments de classe ${ }^{31}$. L'enquête de Claude Le Du, auprès de 130 informateurs des Côtes-du Nord entrés au CP dans la première moitié du $\mathrm{XX}^{\mathrm{e}}$ siècle, confirme la permanence de cette situation de diglossie. Comme le dit laconiquement un informateur : "souvent la rentrée se faisait à Pâques. Ils avaient cinq ans. Ils parlaient exclusivement breton ${ }^{32}$.

Deux autres facteurs liés aux difficultés du milieu sont relevés par les inspecteurs. Ils concernent d'abord la fréquentation scolaire et les conditions d'accueil

26 Fanch Broudic, La pratique du breton, op. cit., p. 351.

27 AD Morbihan, 889W2063, rapport d'inspection de Edmond B., février 1918.

28 AD Morbihan, 889W2074, Locuon en Ploërdut, 1923.

29 Pierre Jakez Hélias, Le cheval d'orgueil, Paris, Plon, 1975, p. 230.

30 Jean Rohou, Fils de plouc, Rennes, Éd. Ouest-France, t. 2, 2007, p. 85.

31 Pierre-Marie Mallégol, Miettes de vie aux portes des Monts d'Arrée, Brest, Emgleo Breiz, 2009, p. 93.

32 Claude Le Du, "Les pratiques pédagogiques à l'égard du breton, à l'école primaire publique et privée (1900-1950) ", in Actes du colloque Le français, le breton, l'école, Trévargan, Musée du Parc naturel régional d'Armorique, 1995. 
des élèves. André Quintric a montré le retard important dans la scolarisation des enfants autour de Pontivy. En 1881, 73,8 \% des enfants scolarisables (6-13 ans) fréquentent les écoles de la ville de Pontivy mais dans les cantons ruraux, la proportion est inférieure à $50 \%$. À la même époque, elle est de $85 \%$ à l'échelle nationale. En 1900, malgré l'obligation scolaire, il reste encore $30 \%$ de la population scolarisable des cantons ruraux à ne pas venir à l'école ${ }^{33}$. Les statistiques sur la fréquentation scolaire manquent pour la période qui suit ${ }^{34}$ mais on peut remarquer que ce retard dans la scolarisation reste un leitmotiv des rapports annuels. Au début du XXe siècle, cela concerne les cantons les plus à l'ouest, autour de Gourin, Le Faouët. Pour les inspecteurs, il semble que les parents ne soient pas intéressés par l'envoi de leurs enfants à l'école, quelle que soit cette école d'ailleurs, catholique ou laïque. La situation s'améliore doucement sous l'effet conjugué d'une demande d'instruction et d'une offre poussée par les inspecteurs d'académie, chaque inspecteur primaire devant rendre compte annuellement du nombre d'ouvertures d'école dans sa circonscription. Mais cet effort est fragilisé par les conséquences démographiques de la guerre, surtout dans cet ouest du Morbihan tardivement scolarisé. Ainsi à Gourin, depuis la fin de la guerre, l'absentéisme est important malgré les efforts des maîtres et, en 1924, l'inspecteur y voit une raison essentielle, la forte mortalité des chefs d'exploitation et des ouvriers agricoles, et donc le manque de main-d'œuvre, qui poussent les parents à garder les enfants sur l'exploitation. À Ploërdut, en 1926, l'inspecteur primaire évoque encore le peu d'assiduité, l'indifférence des familles par rapport à l'école et donc, les effets paralysants de cette situation sur l'action des maîtres. Parfois, les élèves viennent, mais très en retard par rapport à l'âge normal, comme à Guilliers, où "les élèves avancés en âge mais retardés par l'instruction deviennent une gêne ${ }^{35}$. Ainsi, dans l'entre-deux-guerres, les écoles de l'ouest du Morbihan continuent d'être considérées comme les plus défavorisées du département, accusant, malgré des contrastes importants entre cantons urbains et cantons ruraux, un retard global dans la scolarisation.

La question des conditions de travail dans l'école est aussi soulevée dans les bulletins d'inspection et dans les rapports annuels. À défaut de statistiques

33 André Quintric, Le développement de la scolarisation..., op. cit., p. 178.

34 En se fondant sur les indications partielles des rapports d'inspection, André Quintric évalue de 7,7 à $15 \%$ les taux d'absentéisme pour des raisons diverses pendant l'année scolaire 1883-1884 dans les cantons autour de Pontivy, (Le développement de la scolarisation..., op. cit., p. 192).

AD Morbihan, 889W2073, rapport d'inspection de Mathurin G., 1922. 
sur la question, on ne fera que constater les annotations régulières sur le manque de place, de mobilier ou simplement sur les classes très nombreuses. Ces considérations ont un fond de vérité mais l'objectif, dans un rapport qui sera présenté au conseil général, est d'abord de souligner la mauvaise volonté des maires à construire les écoles, à les agrandir ou les rénover. C'est ce que souhaite dire l'inspecteur primaire à Cléguérec en novembre 1920 au sujet d'une école de 202 élèves, avec 68 inscrits au CP et seulement 49 présents le jour de l'inspection : "Le cours préparatoire est trop chargé. La disposition des locaux scolaires impose une répartition quelque peu anormale jusqu'au moment où l'école de garçons sera reconstruite". Il précise aussi que la classe est sans feu quand dehors il gèle, et note "Je plains les élèves et le maître. Mettre fin à cette situation ${ }^{36}$. On peut remarquer le lien indéniable entre la hausse progressive mais lente et contrastée de la scolarisation, et l'effort demandé aux communes en matière de construction d'écoles. Dans les villes, les effectifs sont importants et les classes souvent chargées. Dans les zones rurales, les écoles publiques sont peu nombreuses et l'inspection peut maintenir des effectifs très faibles pour conserver l'école ouverte, voire obtenir l'ouverture d'une école de hameau ${ }^{37}$. Il reste que certaines situations peuvent être difficiles : à Plaudren, au mois de mai 1907, l'inspecteur note : "une trentaine d'élèves ne savent pas lire, 15 à 20 ne peuvent pas écrire faute de places aux tables ${ }^{38}$.

\section{2 - Des "commençants" négligés}

Le second facteur de difficulté dans l'enseignement du français est lié aux enseignants eux-mêmes, particulièrement sur la question des "commençants". Elle émerge avec force avec la mise en place d'une section enfantine à l'école élémentaire, comme l'impose l'arrêté du 18 janvier $1887^{39}$, et alors que les écoles normales ne dispensent pas d'enseignement spécifique sur les petits et les débutants en français.

En mars 1887, l'inspecteur d'académie établit un lien entre les difficultés en français dans le département et les pratiques enseignantes concernant les

36 AD Morbihan, 88W2063, rapport d'inspection de Joseph B.

37 André Quintric, Le développement de la scolarisation..., op. cit., p. 187-188.

38 AD Morbihan, 889W2063.

39 La section enfantine devient "section préparatoire" par l'arrêté du 21 juillet 1922. Voir Jean-Michel Chapoulie, "L'organisation de l'enseignement primaire de la III République", Histoire de l'éducation, $\mathrm{n}^{\circ} 105,2005$. 
"commençants". Il constate que la moitié des enfants qui entrent à l'école ne comprennent pas le français, que certes tous les élèves sont occupés mais pas utilement, qu'on ne parle pas assez et surtout qu'" on ne parle point aux commençants". En 1889, une remarque de l'inspecteur de Lorient nous permet de connaitre le sort des «tout jeunes enfants [...], comme il arrivait fréquemment, abandonnés un peu à eux-mêmes pendant leurs premiers mois " ${ }^{40}$. En 1894, l'inspecteur de Pontivy note :

"Une bonne école est celle où tous les élèves, les petits comme les grands, avancent, font des progrès; c'est celle où aucune division n'est négligée. Or le $\mathrm{CP}$ est trop souvent sacrifié ou à peu près $"^{41}$.

En 1918, à Lignol, "le cours préparatoire est délaissé et les élèves sont trop souvent abandonnés à un moniteur ${ }^{42}$. À Ploërdut, en 1919, l'inspecteur recommande de "prévoir une occupation pour les petits" pendant que le maitre travaille avec les grands ${ }^{43}$. Cette impression de prise en charge réduite des débutants est aussi visible dans les témoignages de l'enquête de Claude Le Du. "Nous les petits, on écoutait, on regardait, on ne faisait rien... juste un dessin l'après-midi" dit une de ses informatrices.

Certains inspecteurs sont tellement sensibles à la question des "commençants " qu'ils ont observé qu'une dimension du problème est d'abord de savoir parler aux petits. Ils insistent sur la capacité à parler aux jeunes élèves, avec chaleur et entrain, ce qui amène ces réserves dans les rapports : "il faut plus de chaleur communicative " $"^{44}$, il faut plus de vie et de chaleur communicative $»^{45}$. Ou encore cette formule assez heureuse : "Il n'a ni la fermeté souriante qui convient, ni l'activité joyeuse qui crée l'intérêt et la vie dans un auditoire de jeunes élèves $" 46$. A contrario, les inspecteurs se font un plaisir de mettre en valeur ces qualités quand ils les rencontrent. Tel instituteur a "la douceur qui convient à des petits, la familiarité de bon aloi " ${ }^{47}$, ou bien ils constatent

40 AD Morbihan, T1012, rapport de l'inspecteur primaire de Lorient, 1889.

41 AD Morbihan, T1047, rapport annuel de l'inspecteur primaire de Pontivy, mai 1894.

42 AD Morbihan, 889W2080, rapport d'inspection de Adolphe LD., janvier 1918.

43 AD Morbihan, 889W2081, rapport d'inspection de Armand LD., mai 1923.

44 AD Morbihan, 889W2079, rapport d'inspection de Joseph LB., mars 1905.

45 Ibid., rapport d'inspection de Louis LC., octobre 1903.

46 AD Morbihan, 889W2067, rapport d'inspection de Antoine C., juin 1924.

47 Ibid., rapport d'inspection de Julien D., février 1905. 
"une autorité du maître faite de douceur et de correction" ${ }^{48}$. "Le maître a de l'entrain, il sait parler à de jeunes enfants "49. Ou encore, voici "un maitre qui recherche des moyens nouveaux et des procédés ingénieux pour éveiller, retenir et renouveler l'attention des tout petits ${ }^{50}$.

\section{3 - Un bilinguisme de transition}

D'un point de vue pédagogique, la question qui se pose est celle de l'usage possible du breton pour enseigner le français. Certes, au début de la III République, cette question semble réglée par la formule de l'arrêté du 18 janvier 1887, reprise ensuite dans les règlements intérieurs des écoles : "le français sera seul en usage dans l'école". Mais en réalité, cette décision fait suite à des discussions qui témoignent de "l'attitude ambivalente des responsables éducatifs à l'égard de l'utilisation des langues premières à l'école "51 et qui ont cours tout au long du XIX ${ }^{e}$ siècle.

En 1831 déjà, le ministre de l'Instruction publique Montalivet envisageait un curriculum bilingue proposant pour les débutants une année d'étude de la langue bretonne avec des livres élémentaires traduits du français dans les différents dialectes bretons. Mais le projet est abandonné, face à l'hostilité de deux des trois préfets de la Bretagne bretonnante. Ce qui n'empêche pas le breton de continuer à être le médium principal de l'enseignement dans l'ouest du Morbihan. Sous le Second Empire, plusieurs comptages permettent d'évaluer la place prépondérante du breton dans les apprentissages. Selon l'inspecteur général Villeneuve, en 1863, dans 130 communes sur 230 de la partie bretonnante du Morbihan, l'enseignement se fait en breton, c'est-à-dire que les élèves apprennent à lire et à écrire le breton ${ }^{52}$. L'enquête Duruy de 1864 ne prend pas en compte le même découpage en envisageant le Morbihan dans sa totalité, mais elle semble aller dans le même sens. Elle assure que dans $70 \%$ des écoles du Finistère, et $40 \%$ dans le Morbihan à travers lequel passe la frontière linguistique, les instituteurs recourent aussi bien au breton qu'au

48 AD Morbihan, 889W2073, rapport d'inspection de Mathurin G., janvier 1923.

49 AD Morbihan, 889W2081, rapport d'inspection de Joseph LD., mai 1905.

50 AD Morbihan, 889W2069, rapport d'inspection de Jules D., novembre 1922.

51 Claude Cortier, Laurent Puren, «Français et langues régionales et/ou minoritaires : une mise en convergence difficultueuse", Repères, n 38,2008 , p.63-80.

52 Cité par Fanch Broudic, "La puissante ténacité de l'obstacle de la langue bretonne", in Hervé Lieutard, Marie-Jeanne Verny, L'école française et les langues régionales..., op. cit., p. 190. 
français comme médium d'instruction, utilisent des manuels franco-bretons et font de la traduction la principale méthode d'apprentissage du français ${ }^{53}$.

C'est dans les écoles confessionnelles que le breton est encore le plus utilisé, notamment dans l'enseignement du catéchisme. Dans les milieux catholiques en effet, les tensions avec le pouvoir républicain poussent à la création d'un enseignement plus systématique du breton. Selon Michel Lagrée, le diocèse de Vannes fait figure de précurseur par l'instauration, à partir de 1891 dans les écoles privées du Morbihan, d'un certificat écrit d'instruction religieuse en breton, nécessitant la production d'un matériel pédagogique en breton ${ }^{54}$; ce que déplore la même année l'inspecteur d'académie dans son rapport au préfet. Cependant, ici aussi, le débat est virulent sur les usages de la langue bretonne, et les partisans d'une Église s'instituant en défenseur inconditionnelle du breton s'opposent aux tenants d'un pragmatisme qui vise l'acquisition d'un bilinguisme par les enfants. En 1914, la revue Dihunamb, rendant compte d'un concours de composition en breton organisé dans les écoles catholiques, exprimait cette idée intéressante : "Les enfants soumis à l'enseignement bilingue font bien la différence entre le breton et le français, et évitent d'employer en français des tournures bretonnes et en breton des tournures françaises. Les autres, au contraire, mélangent breton et français, contribuant ainsi, à leur insu, avec les générations qui les ont précédés, à créer cet affreux jargon qu'on entend parfois dans nos villes et bourgs bretons $\|^{55}$.

On ne saurait donc réduire l'usage de la langue à l'opposition entre les écoles en Bretagne, car des écoles catholiques refusent aussi le breton quand, dans l'école publique, on peut voir dans certaines pratiques une forme de pragmatisme ${ }^{56}$ qui se prolonge certainement bien au-delà de la fin du XIX ${ }^{e}$ siècle. Dans l'enquête de Claude Le Du dans les Côtes-du-Nord, quatre informateurs se souviennent que l'institutrice utilisait occasionnellement le breton dans la petite classe ${ }^{57}$. Et Jean Rohou l'a gardé en mémoire : "quand je suis entré à l'école en 1939, l'institutrice s'aidait encore du breton. Elle l'abandonnait

53 Laurent Puren, "Pédagogie, idéologie et politique linguistique...", art. cit., p. 34.

54 Michel Lagrée, Religion et cultures en Bretagne. 1850-1950, Paris, Fayard, 1992, p. 240-244.

55 Cité par Michel Lagrée, Religion et cultures en bretagne..., op. cit., p. 243. Sur le mélange des tournures françaises et bretonnes, voir le petit ouvrage d'Hervé Lossec, Les bretonnismes, Morlaix, Skol Vreiz, 2010.

56 Claude Cortier, Laurent Puren, "Français et langues régionales...", art. cit., p. 63.

57 Claude Le $\mathrm{Du}$, "Les pratiques pédagogiques à l'égard du breton", art. cit., p.97. 
progressivement au cours de la deuxième année ${ }^{58}$. Ce bilinguisme de transition modère sans doute l'idée d'une rupture totale avec la langue maternelle, mais constitue aussi un pis-aller, une béquille pédagogique permettant aux maîtres de se faire comprendre rapidement et non une méthode revendiquée et mise en œuvre de manière systématique ${ }^{59}$. Pourtant, les instituteurs de nos dossiers sont essentiellement issus des parties rurales et bretonnantes du Morbihan, et il y a peu de doute sur le fait qu'ils connaissaient parfaitement le breton. Mais les traces de cette compétence, et plus encore de pratiques de bilinguisme, sont ténues sous la plume des inspecteurs. Elles existent cependant dans le dossier de quelques instituteurs, dont on peut affirmer qu'ils défendent la cause de la langue bretonne. C'est le cas de Julien D. qui, dans son école de Gourin en 1921, a mis au point " un programme d'histoire de la Bretagne annexé au programme d'histoire nationale", ou qui, à Pont-Scorff en 1925, fait comparer les formes française et bretonne de la langue dans l'apprentissage de la composition ${ }^{60}$.

\section{4 - L'absence de méthode d'enseignement du français}

Ces rares références à des instituteurs utilisant le breton ne doivent pas masquer ce qui fut la ligne directrice de l'encadrement scolaire en Basse-Bretagne : l'exigence d'un enseignement exclusivement en français. Pourtant, les inspecteurs ne rejettent pas nécessairement la langue bretonne. Selon l'enquête de 1865, deux inspecteurs sur trois sont originaires des départements bas-bretons et, comme l'indique Gilbert Nicolas, l'accès au corps des inspecteurs primaires est plus ouvert aux bretonnants du fait de leur facilité à communiquer avec les membres des conseils municipaux ${ }^{61}$. C'est aussi pour cette raison que les inspecteurs peuvent valoriser le breton des instituteurs. "C'est un maître tout à fait à sa place à la tête d'une école rurale. Sa connaissance du breton lui est particulièrement précieuse " ${ }^{62}$, note l'inspecteur primaire pour Pierre J., à Guern, près de Pontivy. Le breton est donc un médium utile en dehors de l'école. Mais à l'intérieur, il est nécessaire de l'exclure si l'on veut «rendre un grand service

58 Jean Rohou, Fils de plouc, op. cit., p. 85.

59 Méthode systématisée par quelques maîtres comme Le Gac (mais méthode dérivée de celle de Carré). On peut aussi envisager ce bilinguisme de transition pour nuancer les propos sur l'usage du "symbole", cet objet d'humiliation et de répression linguistique.

60 AD Morbihan, rapport d'inspection de Julien D., 889W2069.

61 Gilbert Nicolas, «Entre attaches régionales et service de l'État...”, art. cit., p. 39.

62 AD Morbihan, 889W2074, rapport de février 1919. 
aux élèves". C'est ce qu'écrit, en 1878, A. Richard, inspecteur primaire de Pontivy. Il faut selon lui amener les enfants "aussi promptement que possible, à pouvoir se servir exclusivement du français pour tous les exercices scolaires ${ }^{63}$. Il ne propose cependant pas de solution et l'idée d'un problème de "méthode" n’apparaît clairement dans les rapports des circonscriptions bretonnantes qu'à partir des années 1886-1887. Constatant, une nouvelle fois, les faibles résultats de l'enseignement du français, l'inspecteur primaire de Pontivy écrit : "Ces résultats sont encore amoindris par les défauts de la méthode, et il faut le dire, souvent par l'absence de méthode " ${ }^{64}$. Dans le contexte breton, cette remarque peut se traduire sous la forme suivante : comment se passer de la traduction dans les échanges entre le maître et ses élèves et surtout dans l'apprentissage de la lecture? Cette question de méthode, ou de son absence, qui semble générale comme nous l'indique André Chervel concernant le renouveau des études de la langue française, prend en Bretagne une dimension particulière, dans la mesure où il s'agit d'abord de se faire comprendre des "commençants".

\section{II - La recherche d'un modèle d'enseignement du français}

Il est nécessaire de s’interroger plus en détail sur ce qui permet de comprendre l'activité d'évaluation mise en œuvre par les inspecteurs dans le contexte des réformes de l'enseignement primaire, portées par les lois Ferry. On observe en effet une modification fondamentale de la conception de l'enseignement du français et, en même temps, l'adoption de mesures particulières pour s'adapter au milieu spécifique de la Bretagne bretonnante. On peut résumer ce changement à travers trois principes, qui réorganisent la perception des inspecteurs de l'activité que les maîtres doivent engager : l'adaptation au milieu, la rapidité des apprentissages et leur simultanéité.

\section{1 - L'adaptation au milieu}

À partir de 1887, une autre tonalité est décelable à Pontivy comme à Lorient dans les rapports annuels, car, expliquent les inspecteurs, "nous avons inauguré cette année dans la région bretonnante des leçons de langage suivant

63 A. Richard, "La langue française et les écoles de la Basse-Bretagne", Revue pédagogique, $\mathrm{n}^{\circ} 6$, juin 1878, p.603-605.

64 AD Morbihan, T1012, rapport de l'inspecteur primaire de Pontivy pour l'année scolaire 1886-1887. 
une méthode rationnelle dans le cours élémentaire " ${ }^{65}$. L'inspecteur de Lorient semble même faire preuve d'un certain enthousiasme :

"Le premier essai a réussi : maîtres et maîtresses se sont résolument mis à l'œuvre et n'ont qu'à se féliciter des résultats obtenus [...] Nous avons fait le premier pas; nous sommes sortis de l'ornière. L'outil est trouvé : reste à le perfectionner ${ }^{66}$.

La clé de ce changement nous est fournie par le compte rendu des conférences pédagogiques de l'année 1887-1888. Dans l'arrondissement de Pontivy, elles ont été consacrées à «l'enseignement de la langue française en pays breton". On connaît les questions posées aux instituteurs : "Moyens à employer pour apprendre rapidement le français aux commençants. Est-il nécessaire de savoir le breton pour enseigner le français en Basse-Bretagne? Quels sont les procédés en usage dans votre classe et quels résultats obtenez-vous? ${ }^{67}$. Selon l'inspecteur, la série de conférences :

"a permis d'attirer l'attention des maîtres sur cette grosse question de l'enseignement du français aux enfants bretonnants, de faire naitre des idées nouvelles sur ce sujet, de combattre la routine en usage dans beaucoup d'écoles et d'indiquer à grands traits la marche à suivre et les procédés à employer" ${ }^{68}$.

Ce qui lui fait dire aussitôt que "l'enseignement de la langue française s'améliore sensiblement", nuançant toutefois son propos en admettant l'existence de "quelques maîtres incapables ou de mauvaise volonté", même si "tous ont adopté une méthode plus rationnelle "69. L’année suivante, l'inspecteur de Lorient revient sur le sujet :

"Une méthode toute nouvelle et rationnelle a été suivie dans l'enseignement du français aux petits bretons. De l'aveu de tous les instituteurs, et ainsi que j'ai pu le constater, les résultats ont été satisfaisants $»^{70}$.

Le nom de l'inspecteur général responsable de la diffusion de cette méthode apparaît peu à peu dans les rapports. En 1890, l'inspecteur primaire de Pontivy, rappelant que les élèves de sa circonscription qui arrivent en classe "ne savent que le breton, on les soumet alors à ce "régime spécial" dont parle Monsieur

65 Ibid., rapport de l'inspecteur primaire de Lorient pour l'année scolaire 1887-1888.

66 Ibid.

67 Ibid., rapport de l'inspecteur primaire de Pontivy pour l'année scolaire 1887-1888.

68 Ibid.

69 Ibid.

70 Ibid., rapport de l'inspecteur primaire de Lorient pour l'année scolaire 1888-1889. 
l'Inspecteur général Carré $\aleph^{71}$. Irénée Carré, inspecteur général depuis 1882 , s'intéresse en effet à l'enseignement du français dans les "milieux difficiles". Il s'inspire des méthodes d'instituteurs alsaciens et des principes enseignés par Charles Vivien à l'école normale de Strasbourg ${ }^{72}$, et popularise ainsi la "méthode maternelle". Il effectue une première mission en Bretagne, terre symbolisant pour lui les difficultés de l'enseignement du français, en 1887, publie un article dans la Revue pédagogique en 1888 et revient en Bretagne en 1889 pour une nouvelle tournée d'inspections et de conférences ${ }^{73}$. Sa méthode est faite pour ces commençants bretonnants qui ont, selon l'inspecteur général, un vocabulaire très restreint, même en breton. Comme il l'écrit et, sans doute le répète-t-il à chaque conférence, les jeunes bretons ont un stock lexical réduit, bien plus que celui des enfants basques, ce qui justifie à ses yeux l'impossibilité d'utiliser la langue bretonne dans la classe ${ }^{74}$. Et lorsqu'ils parlent en français, leurs tournures de phrases sont encore marquées de régionalismes; la prononciation, l'intonation restent celles d'une langue confinée dans l'espace scolaire.

L'inspecteur général et sa méthode sont donc accueillis avec enthousiasme par les inspecteurs primaires du Morbihan, pas seulement par souci d'obéissance hiérarchique, mais parce que ces corps intermédiaires voient dans la méthode Carré une forme de rationalité, à la différence des "procédés incohérents "75 utilisés jusqu'alors. En effet, la méthode est censée reproduire les conditions d'apprentissage de la langue maternelle, en mettant fin à un processus compliqué : écoute des élèves en français, traduction en breton, réponse en breton, traduction en français. Dans la méthode Carré :

"le maitre montre un objet et il le nomme,

les enfants répètent en chœur, puis individuellement, à tour de rôle,

le maître veille à ce que la prononciation soit nette et correcte $n^{76}$.

71 Ibid., rapport de l'inspecteur primaire de Pontivy pour l'année scolaire 1888-1889.

72 Laurent Puren, "Pédagogie, idéologie et politique linguistique...", art. cit., p. 36.

73 "De la manière d'enseigner les premiers éléments du français dans les écoles de la Basse-Bretagne", Revue pédagogique, $\mathrm{n}^{\circ} 2$, février 1888, p.217-236. L'article est bien connu. Voir l'ouvrage de JeanFrançois Chanet, L'École républicaine et les petites patries, op. cit., et les travaux de Laurent Puren, art. cit. et Hervé Terral, "L'école française en Bretagne au regard de la Revue pédagogique", dans Hervé Lieutard, Marie-Jeanne Verny, L'école française et les langues régionales..., op. cit., p. 201-215.

74 Ces propos ne laissent pas indifférents et les critiques sont importantes comme le montre Laurent Puren, art. cit., p. 39.

75 Selon l'expression de l'inspecteur Poitrineau, cité par Jean-François Chanet, L'École républicaine et les petites patries, op. cit., p. 223.

Revue pédagogique, mars 1888. 
L'exercice de langage doit s'inspirer des objets du quotidien et avoir un "aspect mécanique et répétitif" explique Laurent Puren, car selon l'inspecteur général : "ce n'est que par des répétitions fréquentes que les mots se gravent dans la mémoire des enfants. Il faut donc y revenir souvent" ${ }^{77}$, avec des mots et des phrases qui reprennent un modèle, celui du maître ou d'un moniteur. La méthode Carré serait donc "rationnelle" parce qu'elle permet aux instituteurs de s'adapter aux jeunes élèves monolingues. Quant au contenu des leçons, l'adaptation consiste à penser l'école bretonne comme une école rurale, en mettant en avant une approche "simple, clair [e] et vivant [e]", intégrant le monde qui entoure le jeune élève scolarisé. Comparant les choix d'un sujet de causerie faits par deux enseignants, l'inspecteur de Lorient, au regard de celui intitulé "chanson de mai" dont l'institutrice justifie le caractère poétique, explique combien il préfère "le sujet fort peu poétique donné par un instituteur voisin "le cochon domestique, les services qu'il nous rend” ". L'inspecteur d'académie commente : "ce n'est pas que nous voulons bannir la poésie, loin de là! Mais soyons d'abord pratique »78.

Soyons aussi rapide, sommes-nous tentés de dire, tant à ce moment les inspecteurs primaires des circonscriptions bretonnantes doivent établir des relations entre les modalités d'adaptation au milieu et la réorganisation de l'enseignement du français au niveau national. L'accélération de l'apprentissage de la lecture devient une question essentielle.

\section{2 - La rapidité des apprentissages}

On peut même parler d'un forçage des apprentissages, qui rend compte d'une transformation radicale de la conception de l'enseignement du français. AnneMarie Chartier a montré que, jusqu'à la mise en place de l'école républicaine, domine une conception élaborée par les Lassaliens qui vise à ralentir les apprentissages. La finalité de l'école, pensée par Jean-Baptiste de La Salle, est "l'instruction des choses du salut, c'est-à-dire la catéchisation, l'inculcation des pratiques religieuses et l'acquisition de comportements moralisés " ${ }^{79}$. Comme les "parents ne demandent qu'un savoir-lire-écrire élémentaire et retirent les enfants dès qu'il est acquis, il faut faire durer cet apprentissage assez longtemps pour construire une "éducation", c'est-à-dire un véritable habitus scolaire chrétien

77 Laurent Puren, art. cit., p. 45. Voir notamment les critiques de Perrin sur la méthode Carré.

78 AD Morbihan, T1012, rapport de 1890.

79 Anne-Marie Chartier, L'école et la lecture obligatoire, op. cit., p. 64. 
capable de résister au temps ${ }^{80}$. Dans l'école républicaine, les inspecteurs ont au contraire l'obsession du temps, la volonté incessante de raccourcir celui des apprentissages fondamentaux, et notamment de la langue française.

"Il faut que l'enfant connaisse le plus tôt possible la langue de son maître et de ses livres", explique l'inspecteur primaire de Pontivy en 1888. Et dans son rapport, l'année suivante, il intègre cette dimension à la question posée aux enseignants lors de ses conférences pédagogiques : "quels sont les moyens à employer pour apprendre rapidement le français aux commençants? ${ }^{81}$. On retrouve cette dimension temporelle dans de nombreux rapports d'inspection. À un instituteur, on recommande en mai de "hâter la marche de la deuxième section" des enfants du CP entrés à Pâques! D'un autre, on juge les progrès "assez rapides". À un autre encore, dont la première division "déchiffre assez vite", l'inspecteur indique de "passer de la syllabation à la lecture courante". Ce type de remarque revient dans plusieurs rapports : "ne pas s'en tenir à la lecture mécanique", "bannir la syllabation pour arriver le plus tôt possible à la lecture courante». Elle est justifiée par le fait que les élèves ne peuvent commencer d'autres apprentissages en français qu'à partir du moment où leur compétence en lecture est suffisamment établie. C'est l'inspecteur de Pontivy, circonscription où les progrès sont jugés bien lents, qui résume les enjeux de ce "forçage" de l'apprentissage de la langue française :

"Si l'enfant met deux ou trois ans pour apprendre à lire, il sortira de l'école presque aussi ignorant que lorsqu'il y était entré. L'instruction proprement dite ne commence réellement, en effet, qu'au cours élémentaire. Le temps que l'enfant passe au cours préparatoire est pour lui une période de préparation, d'initiation. Cette période est souvent trop longue : nous avons recherché les moyens pratiques de l'abréger et d'amener l'enfant à suivre le plus tôt possible le cours élémentaire „ ${ }^{82}$.

Lire et parler en français sont des conditions premières et donc urgentes pour permettre une poursuite de la scolarité et l'entrée dans "l'instruction". Il ne faut sans doute pas opposer trop rapidement la lenteur de "l'éducation" qui est au cœur de la conception des Lassaliens à "l'instruction" des républicains. Néanmoins, dans le contexte de lutte des deux écoles, ces deux concepts peuvent rendre compte d'oppositions autant idéologiques que fonctionnelles.

80 Ibid.

81 AD Morbihan, T1012, rapports annuels de 1888 et 1889.

82 AD Morbihan, T1047, rapport de 1894. 
Le forçage des apprentissages donne d'ailleurs des résultats que l'inspecteur d'académie, en visite dans les cantons éloignés de Gourin et du Faouët, vient vérifier en constatant "véritablement émerveillé", "l'aisance avec laquelle certains enfants amenés en classe quelques mois auparavant" répondaient à ses questions en français ${ }^{83}$.

\section{3 - La simultanéité des apprentissages fondamentaux : le lire/écrire}

Le concept de simultanéité recouvre la nécessité de penser les actions de lecture, d'écriture et de langage dans leur complémentaritée ${ }^{84}$. L'arrêté organique du 18 janvier 1887 ne dit rien de cet apprentissage simultané, mais il énumère les types d'exercices constitutifs de l'enseignement du français : "exercices de lecture, lectures expliquées, leçons de grammaire, exercices orthographiques, dictées, analyses, récitations, exercices de composition, etc. ${ }^{85}$. Il n'apporte aucune précision sur les manières d'organiser et d'utiliser ces exercices dans le travail avec les commençants. En 1894, le rapport de l'inspecteur primaire de Pontivy propose donc une adaptation de ces exercices au CP :

"1- Les exercices de langage sont indispensables pour apprendre un peu de français à des enfants qui ne savent que le breton. Le maître doit faire parler beaucoup les enfants.

2- Les leçons de lecture se feront collectivement aux tableaux muraux ou au tableau noir et non individuellement au livret de lecture comme cela se pratique dans quelques écoles.

3- Faire marcher de front lecture/écriture et premières notions d'orthographe usuelles.

4- Apprendre à lire les lettres imprimées et manuscrites puis s'exercer à les reproduire sur l'ardoise ou le cahier; puis sans modèle sous la dictée, puis appliquer la méthode à la syllabe et enfin au mot» ${ }^{86}$.

En 1913, l'inspecteur insiste sur le fait d'orienter résolument les efforts vers l'enseignement du français dans les petites classes en pays breton. "En réalité tous les exercices à l'emploi du temps doivent converger vers ce but : d'abord les exercices de langage, puis les leçons d'écriture, de lecture ${ }^{87}$. Et

83 AD Morbihan, T1048, rapport de 1901.

84 Voir Anne-Marie Chartier, L'école et la lecture obligatoire, op. cit., sur les changements des années 1860 pour les générations qui ont appris à lire et à écrire en même temps.

85 Dans André Chervel, Histoire de l'enseignement du français, op. cit., t. 2, p. 128.

86 AD Morbihan, T1047, rapport de l'inspecteur primaire de Pontivy, 1894.

87 AD Morbihan, 889W2073, rapport d'inspection de Louis G., janvier 1913. 
si les instructions sur les nouveaux programmes du 20 juin 1923 attribuent rétrospectivement à l'arrêté de 1887 la nécessité "d'unir étroitement l'enseignement oral et le travail écrit", il nous semble qu'elles insistent davantage sur la combinaison des leçons de lecture avec celles d'écriture : "les deux enseignements sont solidaires, et il y a souvent intérêt à les donner simultanément ${ }^{88}$.

Adaptation au milieu, rapidité et simultanéité des apprentissages, voilà donc le nouveau cadre organisateur des inspecteurs primaires dans leurs évaluations et dans l'orientation des conseils qu'ils ne manquent pas de prodiguer quotidiennement. Il leur reste maintenant à favoriser de nouvelles pratiques pédagogiques.

\section{III - Favoriser d'autres pratiques}

D'où peuvent provenir les nouvelles pratiques? Certainement pas de formes de bilinguisme telles que certains témoignages ou enquêtes ethnographiques ont pu les rendre visibles. L'analyse des rapports d'inspection montre au contraire que les méthodes promues dans le cas des élèves monolingues bretons trouvent leurs ressources dans les différentes évolutions de l'enseignement primaire entre 1882 et 1940, à partir de la loi de 1882 et de l'arrêté organique de 1887 d'une part, à partir des instructions sur les nouveaux programmes des écoles primaires de 1923 d'autre part. Ces deux textes nous permettent de mettre en évidence deux temps autour de l'année 1923, un avant et un après, qui correspondent à la possibilité de résoudre deux problèmes professionnels.

Avant 1923, les rapports des inspecteurs montrent qu'ils cherchent à résoudre la manière de passer d'une culture quasi exclusive de l'écrit à un usage massif de l'oral. Après 1923, leurs préoccupations s'étendent à une prise en compte par l'enseignant de l'activité de l'élève, comme le prescrivent alors les instructions. Il n'existe cependant pas de césure franche. Les inspecteurs continuent après 1923 d'insister sur les avantages des exercices de langage. Et parfois, avant 1923, une intuition semble ouvrir sur d'autres perspectives pédagogiques. Mais globalement, le regard et l'analyse des inspecteurs sont orientés par les notions à leur disposition à cette époque : "l'enseignement oral» d'une part, puis après la Première Guerre mondiale, et les instructions de 1923 assurément, "la méthode active".

Dans André Chervel, Histoire de l'enseignement du français, op. cit, p. 323. 
Les rapports indiquent des directions pédagogiques, proposent des pistes à suivre, nous font voir des difficultés dans les descriptions qu'ils opèrent des situations rencontrées. Ils mettent en valeur les problèmes de cet enseignement du français aux jeunes bretons et les différents éléments qui les constituent.

\section{1 - Le passage à "l'enseignement oral"}

"À mon arrivée dans l'arrondissement, j'ai été frappé du grand nombre des leçons et des exercices de grammaire, des dictées, des conjugaisons et des analyses écrites imposées à de pauvres enfants sous prétexte de leur apprendre le français. On aurait pu ainsi leur apprendre l'orthographe et la grammaire s'ils l'avaient comprise, mais le français jamais. J'ai insisté [...] sur cette partie fondamentale des programmes et aujourd'hui les leçons de grammaire commencent plus tard, les analyses écrites sont moins nombreuses, les dictées moins longues et mieux choisies, l'enseignement oral a pris le pas sur les devoirs écrits; il est même exclusivement employé au début" ${ }^{89}$.

Il faut être prudent avec ce genre de déclaration, coutumière dans les rapports annuels, mais qui a cependant l'intérêt de mettre en valeur la rupture recherchée dans les pratiques professionnelles des maitres avec la promotion d'un "enseignement oral». C'est en effet désormais la clé de voûte de l'enseignement du français aux jeunes monolingues. Ils "parlent avec beaucoup moins d'hésitation que s'ils avaient appris le français par des traductions, car c'est dans cette langue qu'ils formulent d'abord leurs idées " ${ }^{90}$. Dans ce contexte, les inspecteurs tentent à leur tour de préciser les contours de nouvelles pratiques :

"Je ne cesse de conseiller aux instituteurs et institutrices de remplacer les longs et monotones exercices de lecture machinale par des résumés, des conversations amenées par la lecture d'un texte simple et facile. Pour moi, dans nos écoles bretonnes, voici comment cet exercice doit être compris :

- lecture par le maître du texte choisi

- lecture simultanée du même texte par les élèves de la division

- lecture individuelle par un certain nombre d'élèves

- résumé du texte lu et conversation se rapportant au texte ${ }^{91}$.

Contre les procédés qui organisent l'apprentissage à partir de l'écrit, ces propositions veulent au contraire mettre en avant l'oral en donnant la priorité

89 AD Morbihan, T1012, rapport de l'inspecteur primaire de Pontivy pour l'année 1886-1887.

90 Ibid., rapport de l'inspecteur primaire de Pontivy pour l'année 1887-1888.

91 Ibid., rapport de l'inspecteur primaire de Lorient pour l'année 1886-1887. 
à la lecture et aux exercices de langage. Il faut faire en sorte que les "méthodes routinières", essentiellement les longues copies et la lecture collective sans intervention du maître, disparaissent. Mais cela pose le problème d'une autre forme d'activité du maître qui ne s'improvise pas et dont les techniques ne s'exercent pas facilement. Très tôt, certaines remarques ont mis en avant les difficultés de certains maîtres dans cette conversion vers plus d'oral : "l'instituteur ne sait pas expliquer la lecture" (1885), "les leçons orales sont rares et mal faites" (1887), "jamais de leçons orales" ou "les enfants ne parlent pas", "l'enseignement est trop routinier, c'est la vieille méthode de récitation des élèves par les élèves au lieu de l'interrogation par le maître" (1899) ${ }^{92}$. Les conseils révèlent aussi en creux les mêmes difficultés : "faire des exercices de langage pour les habituer à former de petites phrases bien construites", "partir de petites causeries" $(1903)^{93}$, "il faut que les élèves parlent plus et le maitre un peu moins" $(1904)^{94}$. Même date, à Inguiniel : «je recommande le moins possible de lectures collectives", et en 1906 "j'ai noté avec plaisir que les élèves lisent de manière réfléchie " ${ }^{95}$. Et encore, à Billiers, en 1922 : "leçon très monotone et machinale que les élèves savent par cœur et d'où toute intelligence est bannie".

Les inspecteurs mettent aussi en évidence les efforts pour appliquer les méthodes recommandées : à Ligol, en février 1915, l'inspecteur note que le maître "dirige d'après une bonne méthode l'exercice de langage. Une gravure fixée au tableau présente les idées sous une forme concrète; les termes employés sont empruntés au vocabulaire courant et les enfants sont exercés à construire de petites phrases correctes ${ }^{96}$.

En 1917, à Saint-Philibert près d'Auray :

"Bonne étude de vocabulaire. Les termes étudiés sont empruntés à un ordre d'idée assez restreint pour ne pas égarer les enfants. Les mots nouveaux sont écrits au tableau noir et les enfants sont exercés à les faire entrer tour à tour dans une phrase qu'ils comprennent assez correctement. Les progrès sont sérieux en lecture. Le sens du texte simple est bien compris ${ }^{97}$.

92 AD Morbihan, 889W2067, rapport d'inspection de Noël C., décembre 1899.

93 AD Morbihan, 889W2074, rapport d'inspection de Amédée H., mai 1903.

94 AD Morbihan, 889W2071, rapport d'inspection de Alexis G., avril 1904.

95 AD Morbihan, 889W2068, rapport d'inspection de Emile C., avril 1904 et février 1906.

96 AD Morbihan, 889W2080, rapport d'inspection de Adolphe L., février 1915.

97 AD Morbihan, 889W2063, rapport d'inspection de Edmond B., mars 1917. 
D’Alexandre B., jeune instituteur installé à Plouray, près de Gourin, l'inspecteur écrit, lors de son examen de stage en mai 1922, qu'il a "une connaissance déjà sûre des méthodes et procédés applicables à l'enseignement dans une petite classe en milieu breton". L'année suivante, le même inspecteur note que "les exercices de langage sont communs à tous les élèves de la section préparatoire. Ils s'inspirent des exercices de vocabulaire ou leçons de choses et sont conduits par une application logique des principes de la méthode directe ${ }^{98}$. Les rapports d'inspection valorisent les bons procédés qui permettent les apprentissages simultanés de la lecture, de l'écriture et du langage, comme à Camors en 1922 :

"J'assiste à mon entrée en classe à un exercice de langage sur le sujet suivant : le printemps - quelques fleurs du printemps. Je remarque que les élèves suivent attentivement la leçon et que le maître, d'ailleurs en présentant des fleurs à ses auditeurs, les intéresse à leur observation et leur description. Chemin faisant, on étudie les termes nouveaux, écrits à mesure au tableau noir, on les introduit dans de petites phrases que les élèves, guidés par le maître, s'efforcent de bâtir correctes. Et l'exercice oral se continue par un devoir d'application sur l'ardoise. Chaque élève, à l'aide des jalons posés au tableau pendant l'exercice oral, reconstitue les phrases étudiées. La leçon, satisfaisante comme méthode, se révèle probante par les résultats "99.

Le rapport insiste sur la méthode, mais il rend compte aussi de l'implication du maître dans le travail de la classe. Du point de vue des pratiques, c'est une rupture fondamentale qui est recherchée. L'enseignant présente les éléments de la leçon, les écrit au tableau, "guide" les élèves. Les remarques de l'inspecteur primaire à Guilliers, en 1924, accentuent encore la valorisation de cette activité :

«Écriture au cours préparatoire : les cahiers des élèves sont tout préparés. Sur chacun d'eux figurent les mots modèles écrits de la main du maitre en rouge et se rapportant à la leçon de lecture qui précède. Le maître veille attentivement à la tenue du corps, du porte-plume, et ne quitte les élèves que l'exercice en bonne voie. C'est très bien. À noter enfin que les mots modèles des cahiers ont fait l'objet, en guise de préparation, d'un bref mais suffisant exercice de lecture collectif et individuel. Ainsi M. G. a heureusement associé la lecture des caractères imprimés, la lecture des caractères manuscrits et la dictée des sons. Toute la méthode de M. G. est marquée par cet effort qui tend à réaliser l'unité de direction de l'enseignement à la faveur d'exercices de formes variées mais appliquées au même objet ${ }^{100}$.

98 Ibid., rapport d'inspection de Alexandre B., mai 1922 et décembre 1923.

99 AD Morbihan, 889W2069, rapport d'inspection de Charles G., avril 1922.

100 AD Morbihan, 889W2073, rapport d'inspection de Mathurin G., décembre 1924. 
Ici, l'instituteur semble réussir l'idéal d'intégration des apprentissages. Mais on observe aussi l'importance de l'engagement de l'enseignant. Le maitre a préparé les cahiers, en déposant, en rouge, son écriture modèle sur chacun d'eux. Il fait attention à la tenue du corps, du porte-plume; son passage dans les rangs engage chaque élève individuellement dans le travail. C'est le début d'une insistance sur le guidage des élèves, sur l'attention aux facteurs de succès, mais aussi sur les "innovations" que certaines visites permettent d'encourager, puis d'en diffuser l'usage. L'inspecteur est attentif à ces gestes professionnels nouveaux, comme à Cléguérec en 1907 : "Le maître fait en ma présence une composition au tableau noir sur les éléments de la lecture. Les sons et les syllabes forment un tableau récapitulatif de ce qui a été étudié. C'est ingénieux et intéressant ” ${ }^{101}$. C'est aussi le cas à Guilliers, près de Plœrmel, en 1922 :

"Je note également l'active curiosité de ce jeune maître qui recherche des moyens nouveaux et des procédés ingénieux susceptibles, par leur forme et leur variété d'éveiller, retenir et renouveler l'attention des tout petits : jeux de dominos, lettres mobiles, etc. J'encourage Monsieur D. à continuer dans cette voie. Les méthodes de "la Maternelle" ont leur place au cours préparatoire" ${ }^{102}$.

À Guern, en 1921 : «Le maître se sert de la méthode phonomimique ${ }^{103}$ pour l'enseignement de la lecture aux commençants. Il en tire des résultats très marqués. Les leçons sont animées, suivies avec intérêt, les progrès rapides et sûrs". L'année suivante, l'inspecteur écrit à l'inspecteur d'académie sur la possible diffusion de la méthode phonomimique et sur la possibilité d'une démonstration par l'instituteur de Guern ${ }^{104}$. Cependant les années passent et les remarques sur la difficulté à modifier les pratiques sont toujours présentes dans les rapports. En 1920, à Guilliers, visitant un maitre tout juste sorti de l'école normale de Vannes, l'inspecteur écrit que les mots du texte n'ont pas été assez expliqués et que les enfants n'ont pas lu individuellement. En 1923, dans la région considérée comme la plus rétive à l'enseignement, entre Guémenée et Le Faouët, l'inspecteur s'agace qu'on "exécute au CP des exercices de copie démesurés". À cela une raison : "Monsieur J. ne possède pas encore la pratique

101 AD Morbihan, 889W2081, rapport d'inspection de Joseph L., décembre 1907.

102 AD Morbihan, 889W2069, rapport d'inspection de Jules D., novembre 1922.

103 La phonomimie est un "procédé d'instruction qui consiste à mettre à côté, non de l'écriture, mais de la parole elle-même, 33 gestes onomatopéiques rappelant à la vue les mêmes idées que les sons et les articulations de la voix rappellent à l'ouïe", Augustin Grosselin, Le Moniteur, 30 mars 1867.

104 AD Morbihan, 889W2081, rapport d'inspection de Pierre L., février 1922. 
de l'exercice de langage, enseignement fondamental en milieu breton "105. Deux ans plus tard, l'inspecteur demande au même instituteur de lire attentivement les directives concernant l'acquisition du vocabulaire en plein milieu breton. Il est vrai qu'il exige aussi que le maître prenne connaissance des instructions ministérielles de 1923 au sujet de l'enseignement du français.

\section{Vers un enseignement plus actif}

L'intérêt porté à l'activité de l'élève constitue un nouveau changement important dans la culture professionnelle des instituteurs. D'un point de vue formel, ce sont les instructions du ministre Léon Bérard, du 20 juin 1923, qui invitent les maîtres à modifier leur position dans la classe : "La seule méthode qui convienne à l'enseignement primaire est celle qui fait intervenir tour à tour le maître et les élèves", dans "un continuel échange d'idées" et en considérant que "le maître part toujours de ce que les enfants savent". Ainsi se définissent les nouvelles règles de la méthode active : "un appel constant à l'effort de l'élève et l'associant au maître dans la recherche de la vérité "106. Elles accompagnent et légitiment l'action des inspecteurs dans leur lutte contre les méthodes machinales : les lectures collectives et leur bruit de fond chantonnant qui ne permettent pas d'entendre vraiment la lecture des élèves, comme à Billiers en 1924 : "un élève lit, les autres répètent, tous les élèves servent ainsi à tour de rôle d'entraîneur et la leçon toute machinale se continue sur le même mode pendant une grande demi-heure avec de rares interventions de Monsieur D. Je ne puis donner à Monsieur D. d'autre conseil que celui-ci : qu'il se mette sérieusement au travail et qu'il apprenne à lire à ses élèves "107. Mais que veut dire "se mettre au travail" ? Une réponse nous est donnée dans un rapport écrit quelques années plus tôt : "suivre de près la lecture collective et individuelle pour rectifier les erreurs " ${ }^{108}$. Ainsi donc, l'activité des élèves ne doit plus être "machinale" mais, au contraire, suivie de façon attentive pour chacun d'entre eux. L'élève doit être écouté pour lui-même, et sa lecture rectifiée autant qu'il convient. L'observation de l'inspecteur va dès lors être centrée sur la manière dont les élèves sont engagés dans le travail de la classe. Dans la circonscription

105 AD Morbihan, 889W2074, rapport d'inspection de Charles J., mars 1923.

106 Dans André Chervel, L'enseignement du français à l'école primaire, op. cit., p. 317-318.

107 AD Morbihan, 889W2068, rapport d'inspection de Alexis D., décembre 1922 et mars 1924.

108 AD Morbihan, 889W2079, rapport d'inspection de Louis L., décembre 1921. 
de Ploermel, l'inspecteur observe en 1923 une pratique qui combine engagement de l'enseignant et activité des élèves, en l'occurrence, les plus petits du CP :

«a. Présentation de la voyelle oi, articulation répétée, collective et individuelle

b. observation des lettres, tracé simulé collectif, tracé individuel au tableau noir et sur l'ardoise

c. formation de mots au tableau noir

d. dictée de sons sur l'ardoise, etc.

Il me suffit de noter les différents points de cette leçon au fur et à mesure de son développement pour conclure : bonne leçon qui retient l'attention de l'enfant par des formes et des procédés variés et tire parti du besoin naturel d'activité des enfants".

Pour la lecture des plus grands, l'inspecteur a une impression plus nuancée : la leçon était trop longue et monotone mais l'exercice tout de même "fructueux en raison de l'application du maître à relever les défauts individuels de lecture "109.

Il s'agit bien d'une redéfinition de l'activité du maître. Avant d'exiger du travail de la part des élèves, le maître doit s'impliquer fortement dans les exercices, ce qui n'est pas sans danger pour ceux qui ne sont pas passés par l'école normale. Il lit, articule, écrit au tableau mais surtout écoute les élèves, corrige la prononciation, fait répéter, modifie les postures d'écriture, fait recommencer, apporte des explications. Les notations des inspecteurs vont désormais dans ce sens, quelles que soient les situations : "Le tableau noir est bien préparé avec grand soin et netteté. Des essais intéressants pour sortir des vieux procédés somnolents et provoquer l'activité individuelle des enfants " ${ }^{110}$. Ainsi, à Lorient, en 1924 : "Les explications ont un caractère très concret où l'action est jointe à la parole" et des "procédés variés destinés à mettre de l'intérêt dans l'exercice». Même le concept d'adaptation en milieu breton trouve à se combiner avec celui d'enseignement actif : "Dans un pays où l'on confond facilement dans la prononciation les "au - ou - un", il y aurait lieu de faire des exercices spéciaux d'articulation avant la leçon, de faire des listes de mots contenant les sons, de les faire classer par les enfants ${ }^{111}$. Et à ceux qui n'auraient pas pris ce chemin, les conseils sont désormais constitués en leitmotiv, comme à Gourin en 1924 : il faut «introduire dans sa pratique professionnelle une méthode plus active qui exige des élèves une participation plus active, à la fois par le geste et par la parole».

109 AD Morbihan, 889W2069, rapport d'inspection de Jules D., décembre 1923.

110 AD Morbihan, 889W2068, rapport d'inspection de Julien D., janvier 1932.

111 AD Morbihan, 889W2063, rapport d'inspection de Joseph A., mars 1924. 
Notre projet était de comprendre comment les inspecteurs primaires, soumis aux injonctions - mais le plus souvent en plein accord avec elles - de mettre en place un enseignement de la langue française sans passer par le breton, ont construit leur discours à destination des maîtres. De par leur position hiérarchique, leurs propos rendent compte d'une pratique et d'un discours d'autorité qui ne peuvent être confondus avec la réalité des pratiques des instituteurs. On peut donc toujours penser que les maîtres n'ont pas appliqué à la lettre l'interdiction de l'usage du breton et qu'ils ont, de façon marginale, comme semblent l'indiquer des témoignages, continué de pratiquer un bilinguisme pragmatique, transitionnel en dehors des visites de l'inspecteur. Il reste qu'on doit accepter cette dimension massive : l'apprentissage du français aux enfants monolingues bretons de l'ouest du Morbihan s'est effectué selon un modèle produit par les acteurs locaux. Ce modèle a articulé deux types de principes. Les premiers visaient à adapter l'enseignement aux particularités du milieu mais sans céder sur l'acte fondateur excluant le breton de l'école, par l'usage de la méthode Carré et son opérationnalisation. Les seconds ont mis en œuvre et combiné toutes les ressources offertes par les programmes et leurs instructions. On peut d'ailleurs parler de co-production d'un modèle tant les textes ministériels ont été traduits, soumis aux enseignants par les inspecteurs primaires, mis en œuvre par certains maîtres dont les bonnes pratiques ont été à leur tour présentées en exemples, formalisées et opérationnalisées par les inspecteurs pour une plus large diffusion. Il est possible de voir dans ce processus une raison structurelle de l'efficacité de l'école en Bretagne à partir du tournant de la Seconde Guerre mondiale.

Yannick LE MAREC Université de Nantes, ESPÉ, CREN yannick.le-marec@univ-nantes.fr 